Provided for non-commercial research and education use. Not for reproduction, distribution or commercial use.

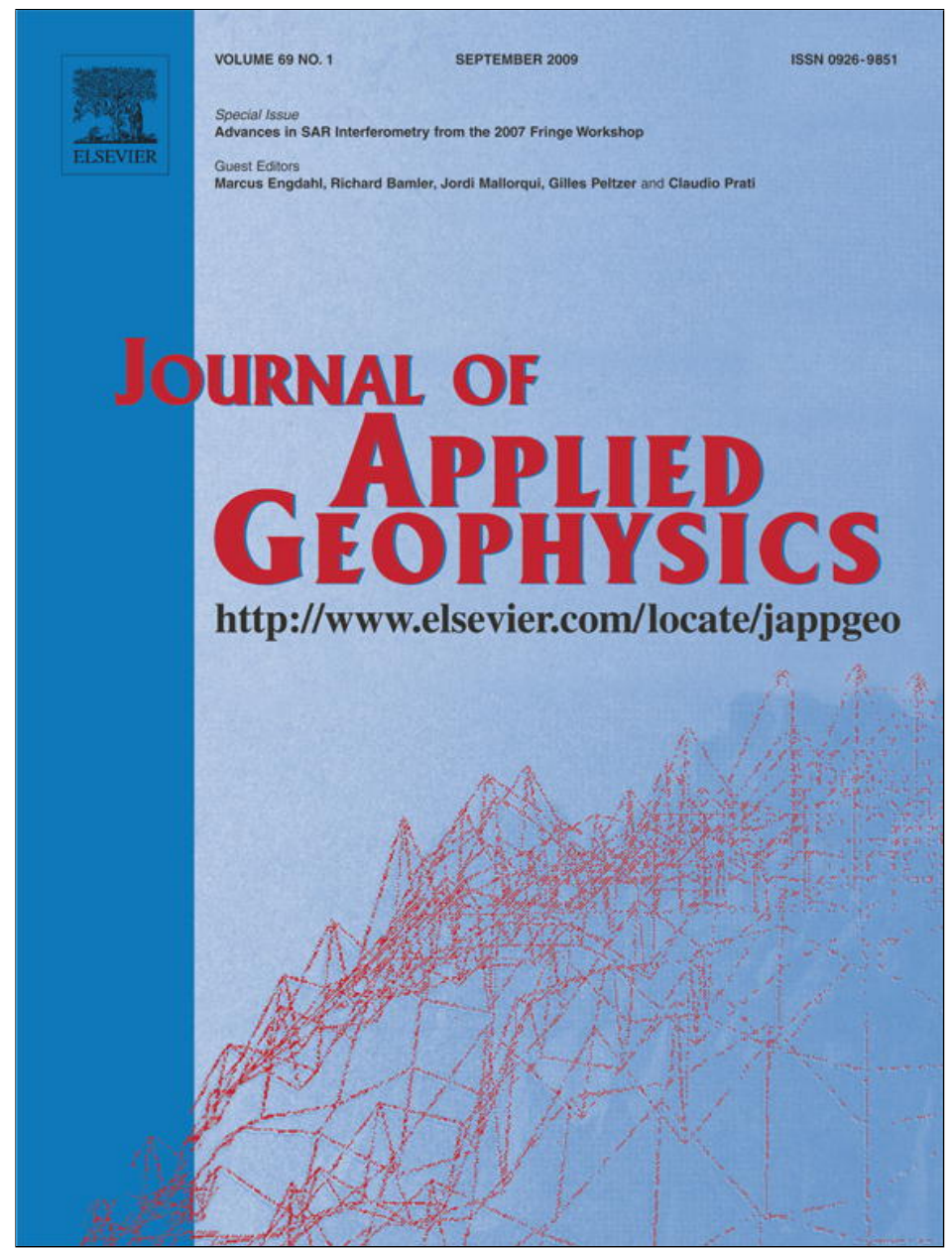

This article appeared in a journal published by Elsevier. The attached copy is furnished to the author for internal non-commercial research and education use, including for instruction at the authors institution and sharing with colleagues.

Other uses, including reproduction and distribution, or selling or licensing copies, or posting to personal, institutional or third party websites are prohibited.

In most cases authors are permitted to post their version of the article (e.g. in Word or Tex form) to their personal website or institutional repository. Authors requiring further information regarding Elsevier's archiving and manuscript policies are encouraged to visit:

http://www.elsevier.com/copyright 


\title{
Practical persistent scatterer processing validation in the course of the Terrafirma project
}

\author{
Nico Adam ${ }^{\mathrm{a}, *}$, Alessandro Parizzi $^{\mathrm{a}}$, Michael Eineder ${ }^{\mathrm{a}}$, Michele Crosetto ${ }^{\mathrm{b}}$ \\ a German Aerospace Center (DLR), Oberpfaffenhofen, Remote Sensing Technology Institute, 82234 Wessling, Germany \\ ${ }^{\mathrm{b}}$ IG, Institute of Geomatics, 08860 Castelldefels, Spain
}

\section{A R T I C L E I N F O}

\section{Article history:}

Received 21 April 2008

Accepted 17 July 2009

\section{Keywords:}

PSI estimation precision

PSI validation

Terrafirma

\begin{abstract}
A B S T R A C T
Terrafirma is an ESA project and a service element in the framework of the Global Monitoring for Environment and Security (GMES) service element programme. Based on the Persistent Scatterer Interferometry (PSI), the project provides a Pan European ground motion hazard information service. The motion monitoring is supplied by commercial companies which act as Operational Service Providers (OSPs). A Product Validation Workgroup (PVW) has been formed for the validation of the motion data products. At the moment, four OSPs operate processing chains for the generation of the basic level 1 product. These take part in a special validation project which intends to demonstrate reliability and accuracy of the PSI motion monitoring.

Two independent and complementary strategies for the validation are foreseen in the validation project in order to drive sustainability. On the one hand, the Product Validation utilizes available ground truth information for the validation and assesses the final geocoded motion data. On the other hand, the Process Validation is a new type of PSI validation and compares the intermediate data in slant range regarding a reference processing and consequently avoids the problems resulting from the geocoding. This newly developed validation approach and the results of the assessment are presented. Essentially, the Process Validation experimentally provides the actual estimation performance for a typical PSI processing based on ERS or Envisat/ASAR stacks. In principle, the lower bound for the deformation deviation an end user can expect ordering a test site processing from different OSPs is reported.
\end{abstract}

(c) 2009 Elsevier B.V. All rights reserved.

\section{Introduction}

Terrafirma is an ESA GMES Service Element project which provides a Pan European ground motion hazard information service. It is based on the Persistent Scatterer Interferometry (PSI) processing which is performed by Operational Service Providers (OSPs). These are commercial companies capable of generating the InSAR-derived motion information. The used processing software is usually in-house developed and the algorithms are often not disclosed. Finally, the generated data products describe terrain motion e.g. subsidence caused by mining, tunnelling, landslides, crustal deformation or volcanic deformation. End users are usually not aware of the processing details and difficulties. However, they expect the processing results delivered by the different OSPs to be technically conformed to agreed standards, validated and to be comparable or even compatible. Terrafirma provides at the moment the steering board to initiate, document and establish such important standard principles for the first time. Therefore, this project brings

\footnotetext{
* Corresponding author. Tel.:+49 8153 281326; fax:+49 8153281420 E-mail address: Nico.Adam@dlr.de (N. Adam).
}

together commercial radar remote sensing companies, national geodetic organisations, end users and governmental research institutions.

In order to achieve a PSI long term validation, a special validation project has been performed. One component of this initial validation effort is the Product Validation (Hanssen et al., 2008) which results in a geo-characterization of the Level 1 data product. It proves the geophysical relevance of the generated data in general. It is based on independent ground truth data and uses geocoded estimation results. Complementary to the Product Validation, the data generation itself is characterized and checked by a Process Validation (Adam and Parizzi, 2007). This check is based on the comparison of the OSP's intermediate processing results with a reference processing. One advantage of the Process Validation is its restriction to slant range geometry measurements. Consequently, problems resulting from geocoding are avoided. E.g., the error propagation during the geocoding may result in a mis-alignment of the persistent scatterers (PSs) between the different OSPs and prevents a direct comparison of the results. Additionally, the validation of the geocoded results regarding independent ground truth data usually requires an interpolation on irregular grid points. The Process Validation has been performed by the German Aerospace Center (DLR) with assistance of the Institute of Geomatics (Spain) which leads the overall validation project. The available data allowed the assessment of the actual performance of 
typical PSI processing chains and the check for systematic effects in the estimation. In order to give an idea of the effort and how the validation is performed the essential aspects of this work are shown at the beginning. Consequently, Section 2 provides the validation principle. In Section 3, the preparations and checks to avoid error propagation and as a result misinterpretations are explained in detail. Section 4 provides the measured deviation between the reference processing and the OSP estimation. Of course, a small discrepancy is expected because the atmospheric phase screen (APS) and noise allow to interpret the data differently. In principle, the PSI estimation algorithm can be differently robust regarding the mentioned phase errors. As a consequence, the OSP dependent error propagation is assessed and the measured deformation spread gives a representative error bound for a typical PSI processing.

\section{Validation principle}

The monitoring of the Earth's deformation effects with mm accuracy by PSI is a powerful but difficult estimation process (Ferretti et al., 1999, 2001). It includes long time span observations using a complex radar sensor, the coherent focusing of the radar acquisitions, the interferometric processing of stacks of radar scenes and the separation of the phase contributions, e.g. deformation, topography, atmospheric effects and noise. Fig. 1 provides a schematic visualization of the signal and noise flow through the PSI estimation subsystems. This processing principle can further be reduced into the standard estimation problem of the estimation of a signal (i.e. deformation) in additive noise.

The signal is the evolution in time of the distance of the stable scatterer on ground to the radar sensor caused by a ground displacement. Noise is added

- by the object phase of the observed overall resolution cell i.e. by uncorrelated clutter,

- by temporal decorrelation of the dominant scatterer and

- by uncompensated multiple scatterers inside a resolution cell.

Also, the APS needs to be considered to be noise in the time series. Its compensation depends on the complexity and robustness of the particular PSI estimator. More measurement uncertainties are introduced by approximations and imperfect algorithms. Examples are the coregistration and interpolation. As a consequence, deviations in the results of the different processing systems can be expected. These can experimentally be measured by comparison with a reference processing. In principle, every processing system which is free of systematic effects can be used as a reference. In the course of this validation, DLR's operational PSI GENSIS system (Adam et al., 2004; Kampes, 2006) has proven to fulfil this requirement and is used.

Finally, the performance of the overall PSI estimation can be characterised by a bias and by the standard deviation of the estimation. A bias would describe a systematic effect and the detection of such is of special importance. It could result from an inappropriate algorithm or simply from an implementation deficiency. The standard deviation results from uncertainty in the measured values and error propagation. It characterizes the actual performance of the PSI estimation. In practice, this is the measurement precision an end user can expect independent from the used processing system or OSP.

Amsterdam and Alkmaar in the Netherlands are the two test sites which are chosen for the comparison. Three data stacks - two from Envisat and one from ERS are processed independently by each OSP in the course of the validation. However, only the best processing (i.e. one test site from the best performing OSP) is finally used to measure the actual estimation precision. This procedure helps to eliminate effects from an un-typical test site, a non-optimal processing system and the system operator (i.e. faulty operation).

\section{Validation preparations}

The validation has been carefully prepared by the project teams. This included for example the selection of the test sites regarding well known subsidence effects (Crosetto et al., 2007). Besides, the temporal and baseline distribution and Doppler frequencies of the radar acquisitions have been assessed. It resulted in a selection of a master scene for each stack and an exclusion of unsuitable scenes. Based on the known subsidence effects in the test site, the processing has been restricted to a linear deformation model. Also, the initial DEM has been defined. In the test site area, the mean height is $43 \mathrm{~m}$ above the WGS84 ellipsoid. This constant value has been used for straightforwardness i.e. to avoid DEM interface problems and alignment problems between the DEM and the radar data. The strong guidelines e.g. the DEM usage, the use of the same scenes and even of one and the same (super) master along with the restriction to a simple deformation model made the processing results mutually comparable. The comparison was supported by a detailed specification of the deliveries and their data format avoiding post processing and conversions.

After the reception of the OSPs estimates, the processed data of all test sites were screened regarding the inter-OSP coregistration which is the basis for the process validation. Moreover, each of the subsystems shown in Fig. 1 was checked for its specific error sources to guarantee correct input data in each subsystem. The result of all these assessments is the selection of the best processing result i.e. of a single OSP respectively processing system and test site to be used in the final comparison with the DLR reference processing. Fig. 2 provides DLR's linear line of sight (LOS) deformation estimation of the finally selected test site Amsterdam. This test site is characterized by a much higher PS density compared to the other Alkmaar test site and by areas with few mm per year subsidence. Figs. 2 and 3 highlight the subsidence areas by the red dots. Especially, Fig. 3 shows in the Google Earth visualization that the deformation areas correspond in their shape to objects as bridges and streets. The following subsections present some examples and provide references for the tests which guarantee valid input data and avoid misinterpretations in the final comparison.

\subsection{Focusing check}

Some OSPs are starting from RAW data. The focusing of these OSPs has been checked in a procedure similar to the interferometric offset

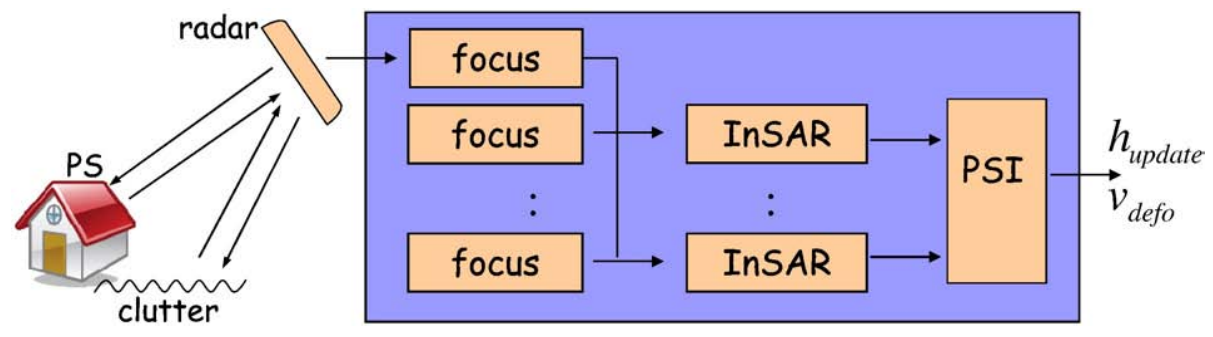

Fig. 1. Signal and noise flow in a PSI processing. 


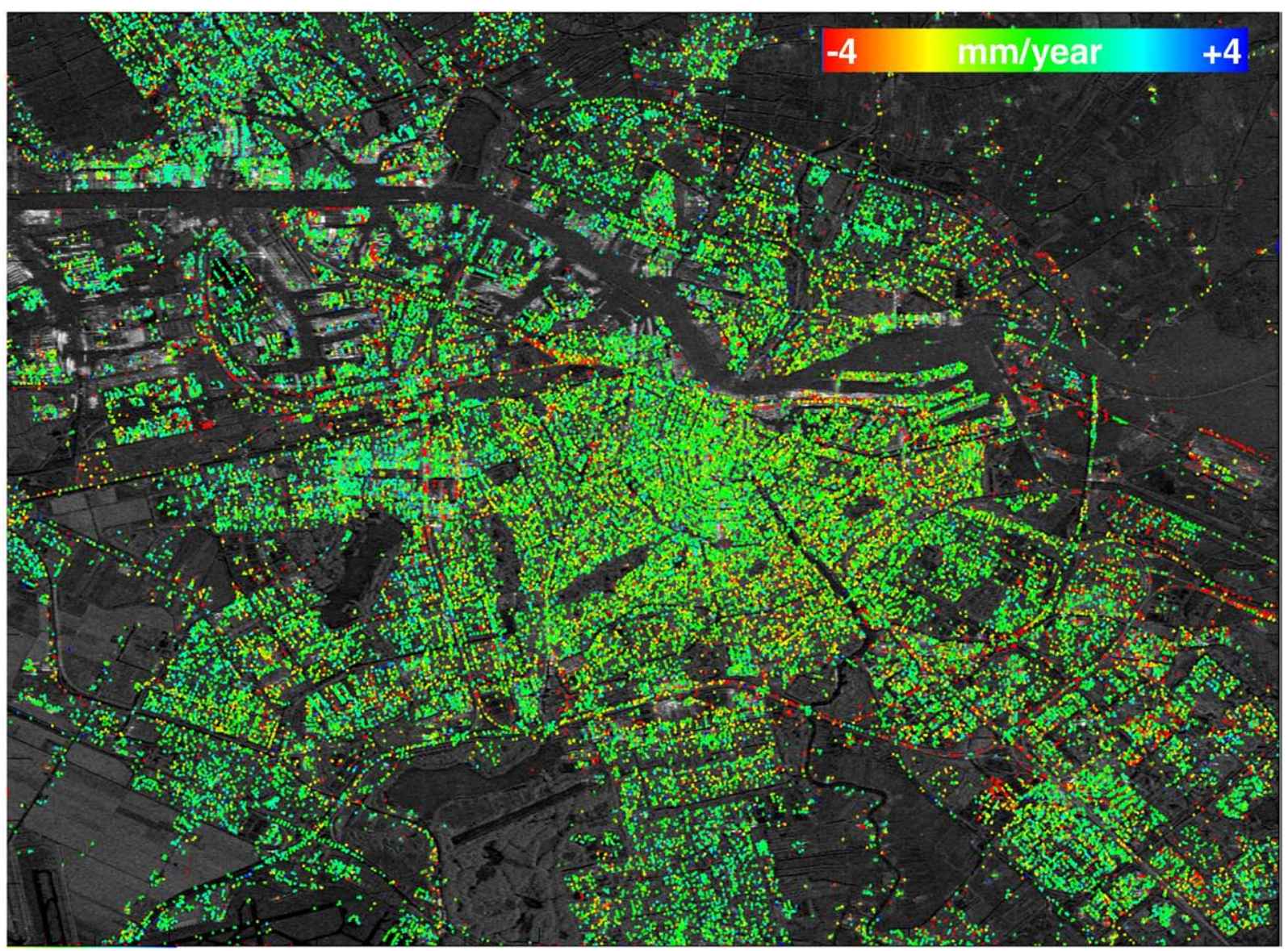

Fig. 2. Linear displacement estimation of the Amsterdam test site. This line of sight (LOS) measurement is the result of the DLR reference processing using 38 interferograms. The red dots characterize areas with about $4 \mathrm{~mm} / \mathrm{year}$ subsidence.

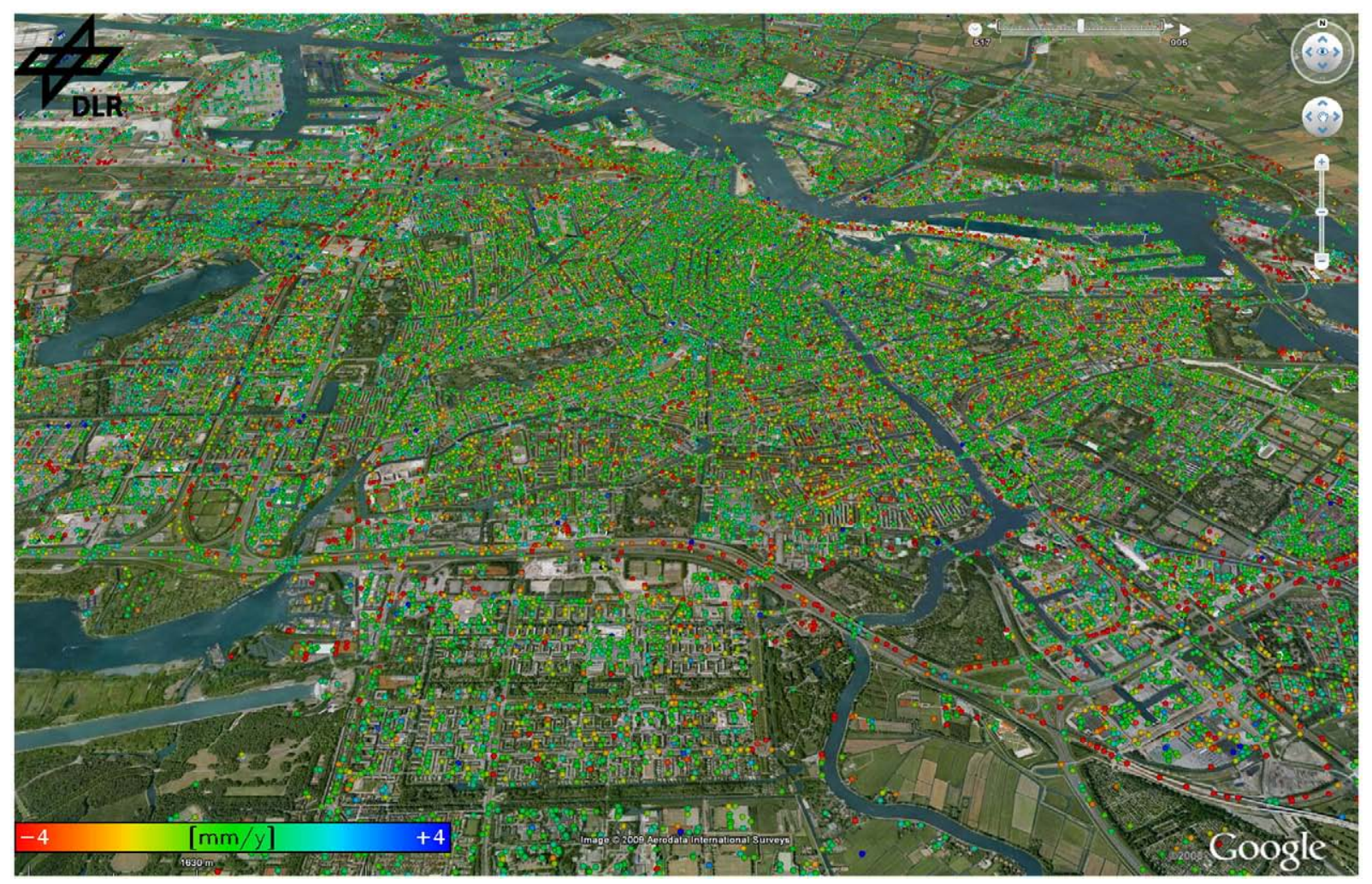

Fig. 3. Geocoded linear displacement estimation of the Amsterdam test site. Notably, the deformation areas correspond in their shape to objects as bridges and streets. 

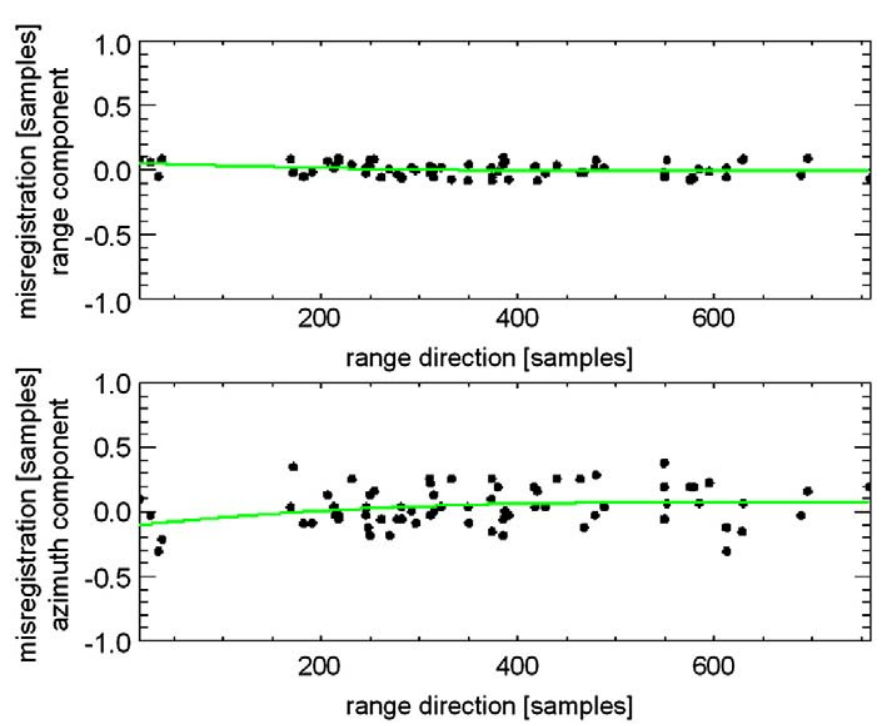

Fig. 4. Upper plot: misregistration in the range component and bottom plot: misregistration in the azimuth component. Both errors are plotted along the slant range direction. The green line describes the misregistration of the respective component of the actual SLC and the spread of the black dots indicates the coregistration precision of the overall data stack.

test (Bamler and Schaettler, 1995; Rosich Tell and Laur, 1996). In this check, SLC scenes processed by ESA are used as the reference. Basically, interferograms combining ESA's and the OSP's focused data are generated. The interferometric phases are checked regarding systematic trends and noise. The smallest phase standard deviation has been measured for the Amsterdam data with 0.38 rad. This can be considered the initial phase error which will propagate into the final deformation estimate. That is, the starting points of the OSPs are different even using one and the same radar data.

\subsection{Coregistration check}

The imperfect coregistration is considered the main error source in the interferometric processing. It was checked for systematic deviations in a few scenes. The applied method is specified in the Terrafirma Quality Control Protocol (Adam and Parizzi, 2008) and is based on a point target analysis (PTA). Essentially, the OSP's data are used without an external reference. I.e. the expected true position of the PSs is estimated by temporal averaging of the scenes amplitudes of a coregistered stack of data which is followed by a PTA in the average image for the high signal to clutter ratio (SCR) point scatterers. Now, every SAR scene of a stack can be checked regarding this reference. I.e. using a PTA, the actual position of the identified PS in a scene is estimated and compared (by subtraction of the range and azimuth component respectively) with the expected location. In doing this, a plot shown in Fig. 4 can be generated. The upper plot shows for a particular scene the coregistration deviation of the range component and the plot below of the azimuth component along the range direction of the master radar scene. The green line is a low order polynomial fit and better describes the systematic error along the range direction. Particularly for this example scene, the systematic error is smaller than $1 / 10$ sample in the range and azimuth component. The spread of the black dots indicates the coregistration precision of the overall data stack. Obviously, the range component (upper plot) is more precisely coregistered compared to the azimuth component (bottom plot).

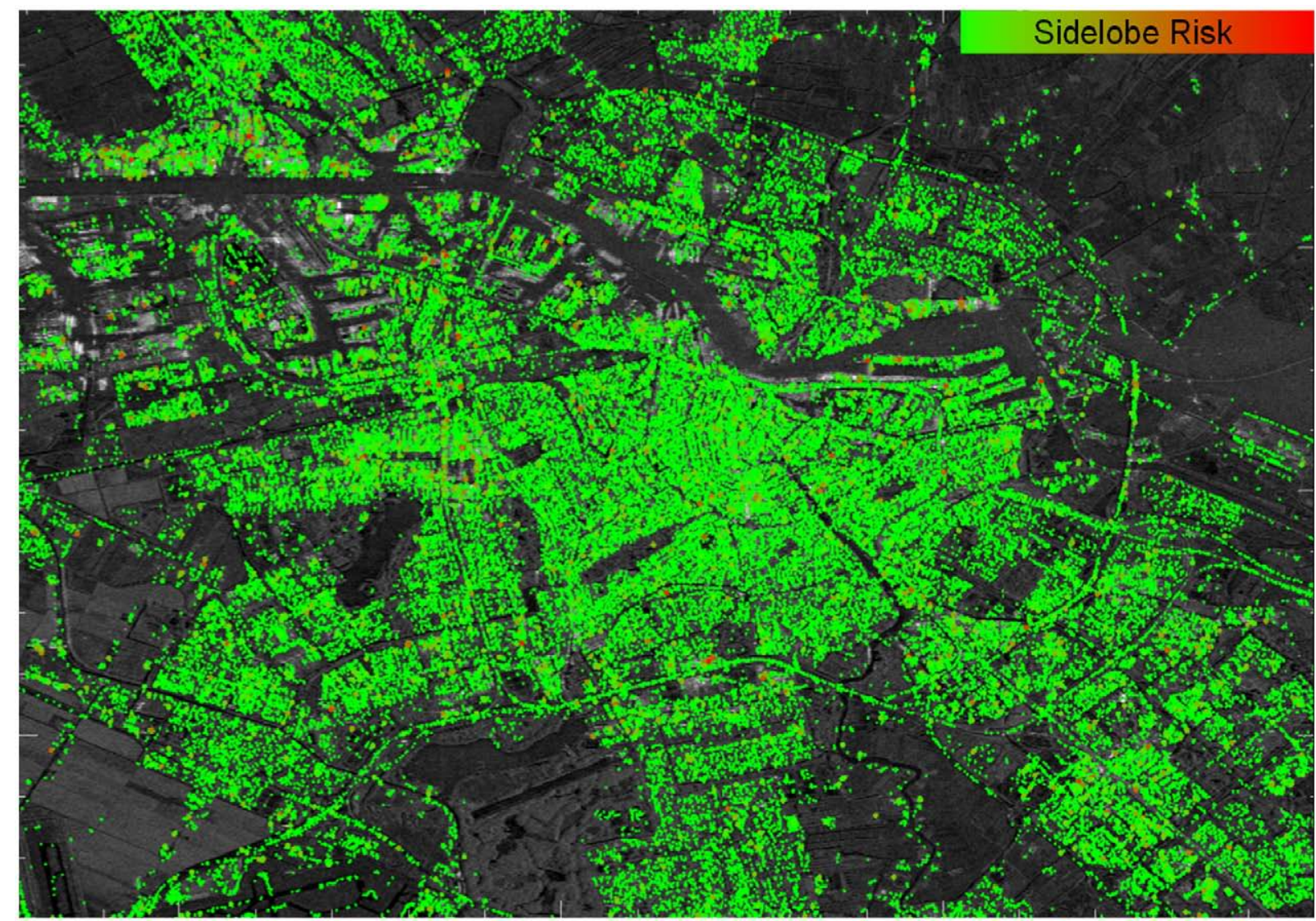

Fig. 5. Overlay of the radar mean intensity image and the detected PS in the Amsterdam ASAR test site. Red indicates detected PS with a high risk to be sidelobes. Finally, the number of risky PS is insignificant and can be excluded to be an error source. 


\subsection{PS detection check}

The PS detection can include wrong scatterers into the estimation process e.g. caused by sidelobes or higher order PS. Both effects were checked using DLR algorithms to detect sidelobes and to detect resolution cells with two dominant scatterers (Adam et al., 2005). Fig. 5 provides an example for the sidelobe risk. Basically, the detection error rate for both effects depends on the thresholds. However, the number of risky PSs in the delivery of the OSPs has been found insignificant resulting in a high reliability in this particular case. Finally, both checks confirmed that the misdetection can be excluded to be a problem in the Process Validation for all OSPs.

\section{Assessment of the estimation precision}

The typical estimation precision is defined as the standard deviation of the deformation measurement the end user can expect exchanging the OSP with its processing system. DLR's validation approach using the PSI GENESIS system as a reference provides this information and clears the question on systematic effects (e.g. biases and algorithmic deficiencies) and excludes these. The assessment finally allows providing a lower bound for the expected deformation estimation error.

The absolute precision of PSI deformation estimates is hard to assess. The comparison with levelling measurements is difficult for several reasons. E.g. it is not always guaranteed that the same objects are observed by levelling and by PSI. In the Process Validation we approach the error assessment from another point of view: We process a given PSI data stack with different estimators (OSPs). The disturbances in the data (atmosphere, noise, decorrelation, orbit errors, and unmodelled motion) give room for interpretation. Different estimators will interpret the data differently, e.g. a median vs. an $L_{2}$ norm estimate. The higher the disturbances, the larger the difference between the estimators will be. We further assume, that the OSPs' algorithms involved in the validation reflect the current state-ofthe-art expert knowledge on PSI data evaluation, i.e. each of the results is "valid". In some sense the differences reflect the inherent uncertainty of the PSI data and method. Hence, the difference (biases and standard deviations) reported in the following can be seen as some best case error of PSI. All the errors can be assumed to depend on the square root of the number in interferograms in the stack. Hence, we normalize our findings to $\sqrt{N}$.

The deformation measurement points (i.e. the PSs) are given at random - but most importantly their quality (i.e. phase stability) varies on a given test site. Nearly ideal scatterers e.g. metal structures like trihedral or dihedral corner reflectors are rarely given. However, the availability of usable scatterers improves if more deterioration in the phase stability is tolerated. The consequence is that the estimation quality varies spatially in a particular test site. Fig. 6 visualizes the relation between the decreasing PS quality and the decreasing measurement precision. Shown are the scatter plots taken from the two independent estimations (but the same data) of deformation measurements for different levels of phase stability of the PSs. The quality of the scatterers decreases from the upper left to the lower right and the random deviation increases indicated by the broadened
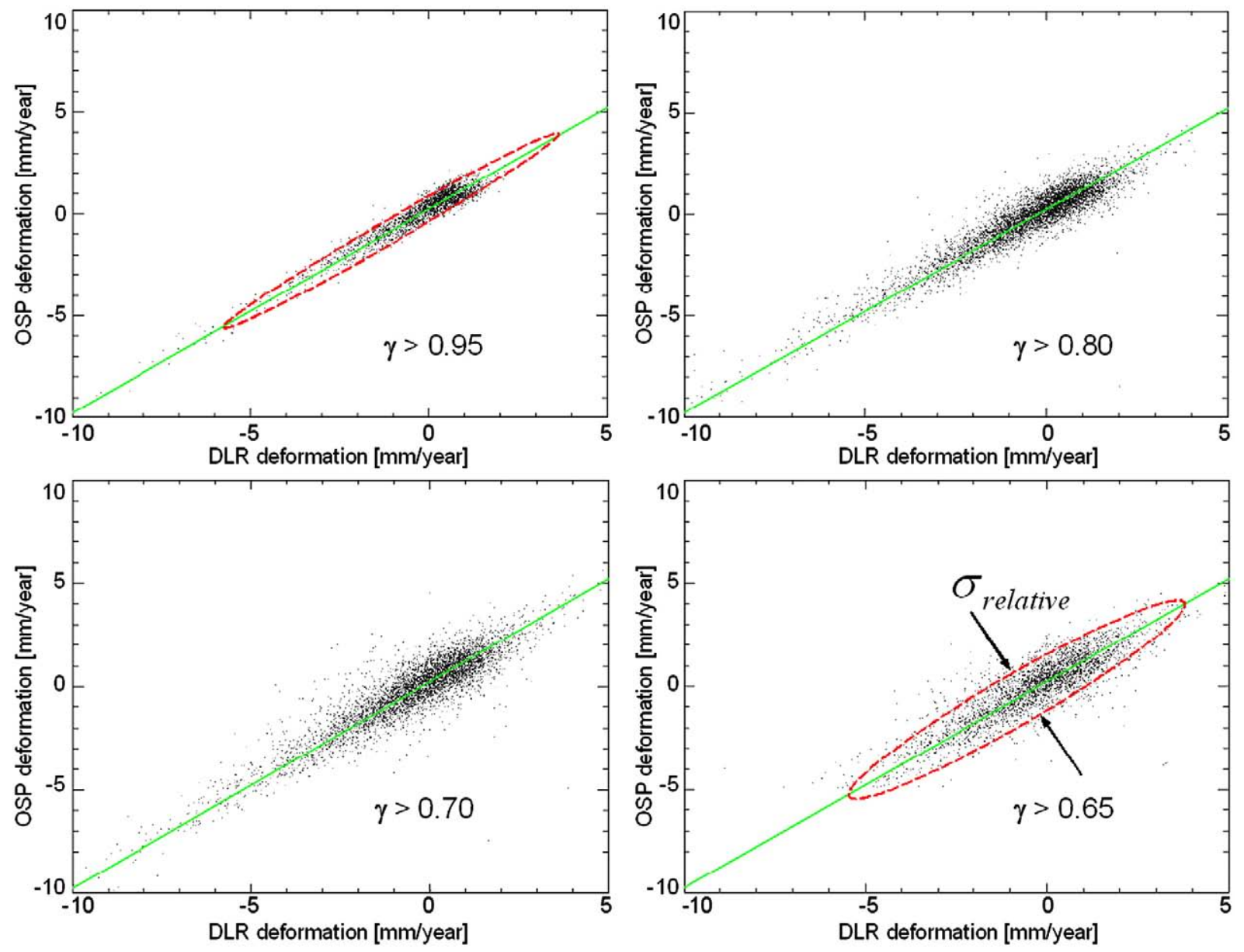

Fig. 6. Visualization of the measured relation between the decreasing PS quality (from top left to bottom right) and the decreasing measurement precision indicated by the broadened scatter plot of deformation estimates from two independent PSI systems. 
cloud of measurements (indicated by the red ellipses). The standard deviation of the deformation estimates is measured by sorting and grouping the estimates according to their coherence. The typical relation (i.e. all OSPs confirm it) between the coherence and the deformation standard deviation is plotted in Fig. 7. 38 interferograms have been used for these results. The best scatterers have a coherence of 0.97 and result in a deformation measurement standard deviation of $0.35 \mathrm{~mm} /$ year, i.e. $2.16 / \sqrt{N} \mathrm{~mm} /$ year. Fig. 7 also provides the number of PSs which are independently detected by the OSP and the reference processing fulfilling a given coherence. These numbers are provided to show that the precision standard deviations are reliably estimated.

The previous precision values are measured on practically available real scatterers. Needless to say, the measured linear relation between the coherence and the deformation standard deviation suggests to predict the estimation precision for optimal scatterers. Such scatterers are described by a temporal coherence of 1.0 which in practice can not be observed. These scatterers need to have an infinite signal to clutter ratio (SCR) and at the same time a linear displacement history over the full observation time span. Basically, the prediction is a heuristic interpretation of the data. It results from a linear extrapolation of the measurements. I.e. it is the standard deviation at the intersection of the extrapolated line and the ordinate at the coherence 1.0. Fig. 7 also visualizes the applied assumption of a linear dependency and the resulting limit in the deformation precision of $1.85 / \sqrt{N} \mathrm{~mm} /$ year.

The previous precision estimates are derived from the two independent PSI processing chains and need to be considered a relative precision. Assuming both processings have the same error contribution, the absolute estimation precision is $1 / \sqrt{2}$ of the measured relative standard deviation. I.e. the best measured absolute deformation standard deviation is $1.53 / \sqrt{N} \mathrm{~mm} / \mathrm{year}$ and the predicted absolute standard deviation for an ideal scatterer is approximately $1.3 / \sqrt{N} \mathrm{~mm} /$ year. Consequently, a lower bound for the PSI estimation error $\sigma_{P S I}$ depending on the number of available interferograms $N$ is given by:

$\sigma_{P S I}(N)=\frac{1.3}{\sqrt{N}}[\mathrm{~mm} /$ year $]$

In conclusion, Fig. 8 provides the lower bound for the typical estimation error depending on the number of scenes. The typical error will be larger as a result of the concept of this assessment i.e. always selecting the best available data. In fact, the given error bound is based

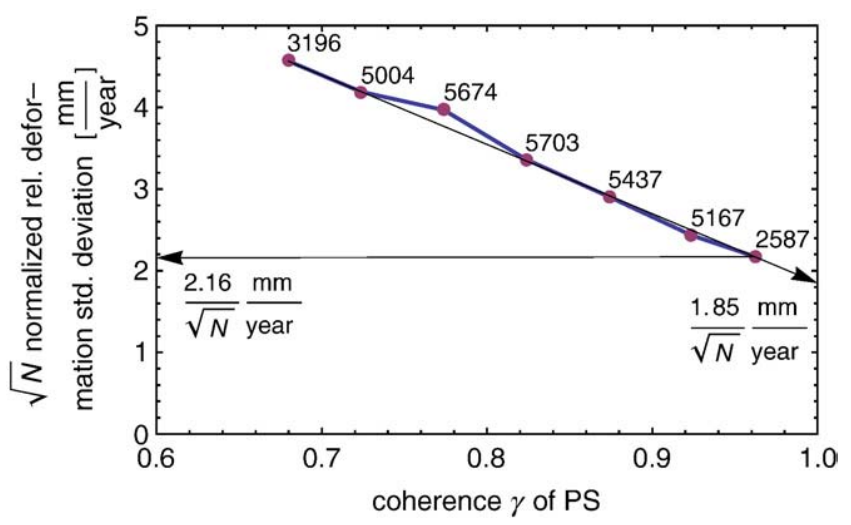

Fig. 7. Typical relation between the PS phase stability (indicated by the coherence) and the measured (purple dots) deformation standard deviation between two independent estimations. The number near the purple dots is the sample size to estimate the std. deviation i.e. the number of PS fulfilling a given coherence. The practically smallest standard deviation is measured to be $2.16 / \sqrt{N} \mathrm{~mm} /$ year. $1.85 / \sqrt{N} \mathrm{~mm} /$ year is the prediction of the deformation estimation precision for a theoretically ideal scatterer. This number results from a linear extrapolation of the measurement.

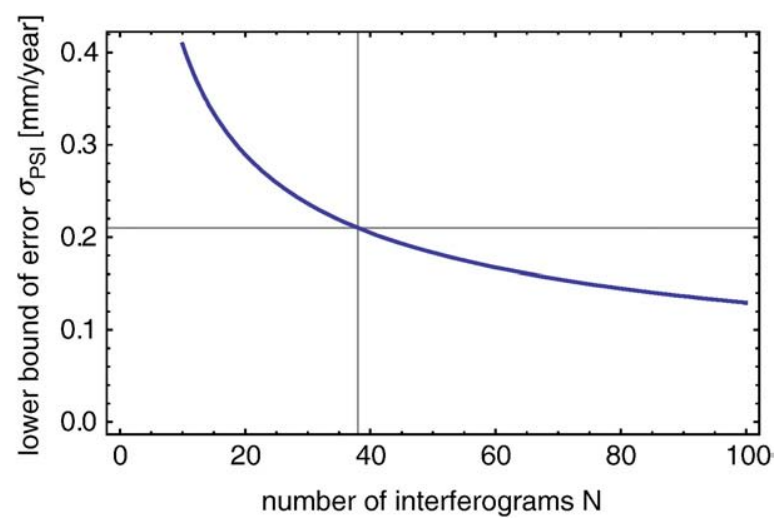

Fig. 8. General lower bound for the estimation precision $s_{P S I}$ which depends on the number of interferograms $N$. The gray cross corresponds to the measured precision for the Amsterdam stack with 38 interferograms. The blue graph is a lower bound of the expected error for ERS like SAR sensors. The typical error will be larger because this analysis is for an ideal scatterer with linear deformation in a test site with a very high PS density and moderate linear deformation.

on an ideal scatterer (i.e. the coherence is one) with linear deformation in a test site with a very high PS density and moderate deformation.

\section{Conclusions}

In the course of a validation project independent PSI results from one and the same test site are compared. This assessment is made straightforward and restricted to slant range and linear displacement rates. Finally, it provides an experimental lower bound for the deformation estimation error for the actual PSI technique over a typical test site using the sensors ERS or Envisat ASAR. In contrast to previous validations, the precision is considered to vary spatially depending on the scatterer's signal to clutter ratio. Basically, the deformation precision depending on the temporal coherence is measured and reported. The heuristic interpretation of the data allows to predict the precision for an optimal scatterer directly from the data. As a result, an ideal scatterer could be measured with a linear deformation standard deviation of $0.21 \mathrm{~mm} /$ year for 38 interferograms. Normalization according to the number of available interferograms allows to provide a more general lower error bound of the PSI processing.

\section{Acknowledgement}

We acknowledge the support by ESA and the PSI intermediate data contribution of Altamira Information, Gamma RS, Fugro-NPA and Tele-Rilevamento Europa (T.R.E.) in the framework of the Terrafirma project. Additionally, we thank Richard Bamler and the anonymous reviewers for the constructive suggestions which greatly helped to improve this paper.

\section{References}

Adam, N., Parizzi, A., 2007. Specification of Validation Approach Part 1: Process Validation, Issue 1.8. online: http://www.terrafirma.eu.com/validation/ValProj/ Validation Rules/Specifications Part 1 Process Validation.pdf.

Adam, N., Parizzi, A., 2008. Terrafirma Quality Control Protocol for Level 1 Products. Issue 1.5, online: www.terrafirma.eu.com/Documents/QCP/QCP_Level1_Products. pdf.

Adam, N., Kampes, B.M., Eineder, M., 2004. The development of a scientific persistent scatterer system: modifications for mixed ERS/ENVISAT time series. ENVISAT/ERS Symposium, online: http://earth.esa.int/workshops/salzburg04/papers_posters/ 2C1_adam_315.pdf.

Adam, N., Bamler, R., Eineder, M., Kampes, B., 2005. Parametric estimation and mode selection based on amplitude-only data in PS-interferometry. Proceedings of FRINGE'05, Frascati, Italy, on CD.

Bamler, R., Schaettler, B., 1995. Phase-preservation in SAR processing: definition, requirements and tests, DLR Tech. Note, Ver.1.0. 
Crosetto, M., Agudo, M., Bremmer, C., Hanssen, R.F., 2007. Technical note on the test site selection: Alkmaar-Amsterdam. online: http://www.terrafirma.eu.com/validation/ ValProj/Site selection technical note.pdf.

Ferretti, A., Prati, C., Rocca, F., 1999. Permanent Scatterers in SAR Interferometry. IGARSS Proceeding 1999, Hamburg, Germany, pp. 1528-1530.

Ferretti A., Prati, C., Rocca, F. 2001. Permanent Scatterers in SAR Interferometry, TGARS, Vol. 39, No. 1, pp. 8-20.

Hanssen, R.F., van Leijen, F.J., van Zwieten, G.J., Bremmer, C., Dortland, S., Kleuskens, M., 2008. Product validation: validation in the Amsterdam and Alkmaar area. online: http://www.
terrafirma.eu.com/validation/ValProj/FinalReports/ProductValidation_report_TNO-TUD. pdf.

Kampes, B., 2006. Radar interferometry- persistent scatterer technique., Remote Sensing and Digital Image Processing, 12, Springer, 211 S., ISBN 1-4020-4576-X. Rosich Tell, B., Laur, H., 1996. Phase preservation in SAR processing: the interferometric offset test, pp. 27-31. IGARSS96, Lincoln, Nebraska, USA. 